\title{
Target Control for Hybrid Systems with Linear Continuous Dynamics
}

\author{
Anastasios Trontis Michael P.Spathopoulos \\ Department of Mechanical Engineering \\ University of Strathclyde \\ 75 Montrose St, Glasgow \\ G1 1XJ, Scotland \\ atrontis,mps@mecheng.strath.ac.uk
}

\begin{abstract}
We consider the target control problem for hybrid systems with linear continuous dynamics. The system is modelled as a hybrid automaton. Control action is applied on the discrete level, while the continuous dynamics is subject to constant or set valued disturbance. The proposed controller ensures that the system can be transferred from any point of an initial set to a target set of the hybrid state space. A control design algorithm based on reachability analysis is proposed. For the implementation of the algorithm approximate reachability analysis is employed. This involves under-approximation of reachable sets under linear continuous dynamics. The algorithm is applied to a batch control problem.
\end{abstract}

\section{Introduction}

The incorporation of discrete dynamics in the modelling of physical systems, which were traditionally modelled by continuous dynamics, has led to hybrid systems. There has been a considerable effort to develop theoretical frameworks and models for hybrid systems. But because of the complex interaction of continuous and discrete dynamics, strong analytical results are not available yet. Besides, computational methods and more specifically reachability analysis are the altematives for efficient analysis and control.

This paper presents a computational method, based on reachability analysis, for the synthesis of control laws for the target control problem [18], [19]: Given a hybrid system, a set of constraints on the evolution of both continuous and discrete states, an initial and a target region in the hybrid state space, do there exist control laws that ensure that the system can be transferred from the initial to the final region without violating the constraints? Control action is considered only on the discrete level, i.e. there is no control on the continuous dynamics. The target control problem is of great practical value. For instance, design problems on process systems that deal with batch material flows [7], [8], [9], [12], can be seen as hybrid target control problems. In such systems material flows go through a number of stages of various treatment, e.g., heating, cooling, mixing, reaction, separation, buffering etc., till the process reaches a desired final state. Then the system is initialised and the next batch goes through the same process.

The target control problem was originally addressed in [12] and mainly in [18] for hybrid systems with integrator continuous dynamics. We extended this initial result to linear hybrid systems in [19], where the continuous dynamics is governed by rectangular inclusions. In this work we make a further extension to hybrid systems with linear continuous dynamics of the form $\dot{x}=A x+u$. The disturbance signal $u$ is either constant or it has a set valued uncertainty. We model the hybrid system using hybrid automata, i.e. finite state machines equipped with continuous variables. The control synthesis is based on the notion of controllability for hybrid automata, as defined in [18], and backward reachability analysis. In the case of integrators or rectangular inclusions, "exact" backward reachability analysis is performed by employing quantifier elimination techniques. However, this not the case for systems with richer dynamics, where in general reachable sets cannot be expressed in closed forms. The only exceptions so far concern some special classes of linear [15] and non-linear [17] vector fields. Hence, for our purposes we resort to approximate reachability analysis. Since our objective is control design, we are interested in under-approximations of backward reachable sets. Although there exist quite a few reliable techniques for overapproximating reachable sets, both for linear and non-linear systems [1], [2], [3], [5], [6], [10], [16], [20], the underapproximation problem has proved to be a lot harder. The results in [11] and [20] are for linear systems and in discrete time only. In this work, we present an under-approximation scheme adopting the over-approximation techniques proposed in [1] and [4].

The paper is organised as follows. In section 2 the hybrid automata framework as well as the target control problem are formally defined. In section 3 we propose the solution to the target control problem as the output of a semi-decidable algorithm. Next, in section 4, we deal with the technicalities of that control design algorithm and namely with the under-approximation of reachable sets. In section 5 our results are applied to a simplified batch control problem. Finally, in section 6 conclusions and directions for further research are given.

\section{Problem Statement}

2.1 Modelling Framework

The hybrid automaton is the basic entity of our analysis.

Definition 1 A hybrid automaton is a tuple $A \equiv(X, Q, I N V, f, T, C, G)$ where:

- $X \subseteq \Re^{n}$ is the continuous state space defining the continuous part of the plant state space $x(t) \in X$.

- $Q$ is the finite set of discrete control locations $q \in Q$. The pair $Q \times X$ gives the global or hybrid state of the system.

- $\quad I N V: Q \rightarrow 2^{X}$ assigns to each control location $q \in Q$ an invariant set $I N V(q) \subseteq X$. The continuous state must lie in this set as long as the system resides in the corresponding location $q$ 
- $f: Q \times X \rightarrow 2^{X}$ assigns to each location a continuous vector field $\dot{x}=A x+u$, with $A \in \Re^{n \times n}$ and $u \in U$, where $U$ is in general a convex polyhedral set in $\Re^{n}$.

- $T=T_{c} \cup T_{u} \subseteq Q \times Q$ is the set of discrete transitions between control locations. $T_{c}$ is the set of controllable transitions while $T_{u}$ is the set of uncontrollable transitions.

- $\quad c: T \rightarrow \mathfrak{R}^{+}$is a cost function that assigns a positive real number to each transition.

- $\quad G: T \rightarrow 2^{X}$ assigns to each $\left(q, q^{\prime}\right) \in T$ a guard set $G\left(q, q^{\prime}\right)$ such that $G\left(q, q^{\prime}\right) \cap I N V(q) \neq \emptyset$. Guards represent the switching conditions that the continuous state must satisfy in order for a transition to take place.

All the sets involved in the above definition are considered closed and compact. Uncontrollable transitions take place as soon as the continuous state enters the corresponding guard set. For that reason the involved guard set $G\left(q, q^{\prime}\right)$ is defined as a hyper-surface on the boundary of the invariant set $\operatorname{INV}(q)$ rather than a full dimensional set in $\Re^{n}$. Uncontrollable transitions model physical requirements, such as safety and security, and we are not allowed to ignore or modify them. They are intrinsic features of the system and we have no control on them. In the case of controllable transitions, the moment that the transition takes place is a design variable. An external system (controller) orders the transition when certain conditions, subject to design, are satisfied. If there is no controller imposed on the automaton, we assume that the transition takes place anytime while $x \in G\left(q, q^{\prime}\right)$.

The introduction of the cost function $c$ in the definition of the hybrid automaton, provides us with greater design flexibility. Having defined transition costs, we can penalise the use of certain transitions. For instance, in a realistic system we may not want to use a sensitive switch or valve very often. So during our design process, by assigning costs to transitions, we take into account this kind of requirement.

Finally, without loss of generality, we assume that there are no resets or jumps in the values of the continuous variables when transitions are taken. Of course the extension of our results to automata that accept reset maps is straightforward at the price of some extra notation.

\subsection{Control Objective}

Given the plant, modelled as a hybrid automaton, and a specification for the desired behaviour our objective is to derive a controller that guarantees the correct evolution of the system dynamics according to the specification. For our purposes, assuming full observability of the hybrid state, the controller is considered to be a map $C: L \rightarrow 2^{Q}$, where $L$ is the set of all control sequences $l(i)=\left(\pi(i), G_{i}^{*}(\pi(i), \pi(i+1))\right) . \pi$ denotes a sequence (path) of control locations $q \in Q$, while $G^{*}$ is the sequence of the control guards. The control guards $G_{i}^{*}(\cdot,) \subseteq G(\cdot$,$) are the design parameters of the proposed$ controller. As long as the continuous state $x$ satisfies the predicate $G_{i}^{*}\left(q, q^{\prime}\right)$ at the location $q=\pi(i)$ and given that $\left(q, q^{\prime}\right) \in T_{c}$, the controller can either order the transition $\left(q, q^{\prime}\right)$ to the next location $q^{\prime}=\pi(i+1)$ or "idle" for some time and order it later. In either case the idling period stops and the controller orders the transition just before the condition $x \in G_{i}^{*}\left(q, q^{\prime}\right)$ becomes false. The idling period reflects design margins. However, in our framework we consider no restrictions over the duration of idling. Following this strategy, the controller "drives" the system along the path $\pi$. Note that there may be the case where $x \in G\left(q, q^{\prime \prime}\right)$ with $q=\pi(i)$ and $q^{\prime \prime} \neq \pi(i+1)$. In this case the controller prevents the transition $\left(q, q^{\prime \prime}\right)$ since $q^{\prime \prime} \neq \pi(i+1)$.

Finally, we cast the target control problem as follows: "Given a hybrid automaton $A$, an initial set $I=q_{0} \times X_{0}$ and a target set $F=q_{F} \times X_{F}$, design the control sequence such that all trajectories initiating from $I$ reach $F$ with the least overall transition cost".

\subsection{Controllability Analysis}

\section{The Solution}

In this section we propose the semi-decidable algorithm for the solution of the target control problem. The key notion behind the algorithm is this of controllability for hybrid automata [18]

Definition 2 An acceptable path $\pi=q_{0}, \ldots, q_{F}$ of a hybrid automaton $A$ is a sequence of control locations, along which there exists a switching strategy such that all trajectories initiating from initial set $I=q_{0} \times X_{0}$ reach the target set $F=q_{F} \times X_{F}$.

Definition 3 A hybrid automaton $A$ is controllable with respect to the initial set $I$ and the target set $F$, iff it has at least one acceptable path $\pi$.

From the above definitions we infer that in order to derive a switching strategy, that drives the system from $I$ to $F$, it suffices to check controllability along paths of $A$ between the initial and the target location. In essence checking controllability along a path $\pi$ is a backward reachability problem. Starting from the target set $F$ we ask to compute the set of states $W$, from which $F$ is reachable by the evolution of the continuous and discrete dynamics. We iterate this computation over $\pi$ till the initial location $q_{0}$ is reached. If $I \subseteq W$ then $\pi$ is acceptable. For the computation of $W$ the following two operators are required.

Definition 4 Given a set $Y=q_{Y} \times X_{Y}$, with $q_{Y} \in Q$ and $X_{Y} \subseteq X$, and a vector field $f$, the continuous predecessor operator pre $2^{Q} 2^{Q \times X} \rightarrow 2^{Q \times X}$ is defined as:

$\operatorname{pre}_{c}(Y)=\left\{(q, x) \in I N V(q) \mid \exists t \geq 0 \forall x^{\prime}=x+\int_{0}^{t} f_{q}(\tau) d \tau:\left(q, x^{\prime}\right) \in Y\right\}$

Definition 5 Given a set $Y=q_{Y} \times X_{Y}$, with $q_{Y} \in Q$ and $X_{Y} \subseteq X$, the discrete predecessor operator pre $_{d}: 2^{Q \times X} \rightarrow 2^{Q \times X}$ is defined as:

$$
\text { pre }_{d}(Y)=\left\{(q, x) \in I N V(q) \mid \exists q^{\prime} \exists x \in G\left(q, q^{\prime}\right):\left(q^{\prime}, x\right) \in Y\right\}
$$

Intuitively, the continuous predecessor operator defines all the states, which can reach a set $Y$ by the evolution of the continuous 
dynamics only. Similarly, the discrete predecessor operator defines all the states that can reach $Y$ by discrete transitions only. After these definitions, the algorithm that performs controllability analysis along a path $\pi$ is the following:

\section{Controllability analysis along $\pi$}

\begin{tabular}{|l|}
$\pi:=q_{0}, \ldots, q_{F}, m:=$ length $(\pi)$ \\
$W_{\pi(m)}:=\operatorname{pre}_{c}(F)$ \\
for $i=m-1$ downto 1 \\
$W_{\pi(i)}:=$ pre $_{d}\left(W_{\pi(i+1)}\right)$ \\
$G_{i}:=W_{\pi(i)}$ \\
$\quad W_{\pi(i)}:=$ pre $_{c}\left(W_{\pi(i)}\right)$ \\
end \\
if $I \subseteq W$ \\
$\pi:$ acceptable \\
end
\end{tabular}

\subsection{Control Synthesis}

Controllability analysis along a path $\pi$, is the main subroutine of the algorithm for the solution of the target control problem. Before presenting its steps we need to define the value $v(\pi)$ of a path $\pi$.

Definition 6 Let $\pi=q_{0}, \ldots, q_{F}$ be a path of a hybrid automaton $A$. Its value $v(\pi)$ is defined by the sum

$$
v(\pi)=\sum_{i=1}^{m-1} c(\pi(i), \pi(i+1))
$$

Hence, the logical diagram of the control synthesis algorithm is depicted in figure 1, while its steps are the following:

Step 1: The algorithm ranks the $K$ shortest paths from the initial location $q_{0}$ to the target location $q_{F}$ in ascending order of value $v(\cdot)$ and stores them in a list. For that reason, a generalisation of the Dijkstra's shortest path algorithm [13],[14] is employed.

Step 2: We check controllability along the first path $\pi=q_{0}, \ldots, q_{F}$ of the list. If the path is acceptable the algorithm terminates successfully. If not, $\pi$ is removed from the list and we proceed to step 3.

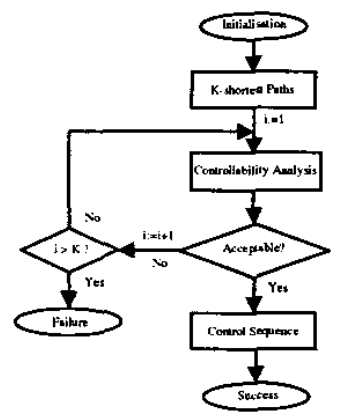

Figure 1: Control Synthesis Algorithm
Step 3: We iterate the steps 2 and 3 for all the identified paths of step 1 . If after testing the $K^{\text {th }}$ path there is no acceptable path found, the algorithm terminates with a negative output.

From a theoretical point of view, if the algorithm terminates in step 3 and no acceptable path has been found, we can not claim that the system is uncontrollable, unless the maximum number of paths in the automaton is $\mathrm{K}$. There may be the case where an acceptable path with rank greater than $K$ does exist but the $K$ shortest paths algorithm naturally does not identify it. Therefore, the proposed algorithm is semi-decidable in the sense that if the system is controllable, it may identify an acceptable path. If not then it gives no answer. Finally, for the control sequence $l(i)$ of the target control problem it holds:

Proposition 1 Given that an acceptable path $\pi$ has been identified, the control sequence for the target control problem is defined as

$$
\begin{aligned}
l(i) & =\left(\pi(i), G_{i}^{*}(\pi(i), \pi(i+1))\right) \\
\text { with } G_{i}^{*}(\pi(i), \pi(i+1)) & =G_{i}, i=1, \ldots, m-1
\end{aligned}
$$

\section{Approximate Reachability Analysis}

The major problem we have to overcome for an efficient implementation of the control algorithm is the computation of the continuous predecessor operator pre $_{r}(\cdot)$. For our purposes, as we stated in the introduction, we need to under-approximate the "backward" reachable sets defined by $\operatorname{pre}_{c}(\cdot)$. For that reason we modify the approximation schemes, proposed in [1] and mainly in [4].

\subsection{Affine Systems}

Let us consider the special class of affine dynamical systems with state equation $\dot{x}=A x+b$, where $A \in \Re^{n \times n}$ and $b \in \Re^{n}$. The set of states from which the target set $X_{f}$ is reachable at time $t$ is defined as:

$$
R_{t}^{-}\left(X_{f}\right) \equiv\left\{x_{0} \mid x_{0}=x\left(t, x_{f}\right), \exists x_{f} \in X_{f}\right\}
$$

where $x\left(t, x_{f}\right)=e^{-A t} x_{f}-\int_{0}^{t} e^{-A(t-\tau)} b d \tau$. The set of states from which the target set $X_{f}$ is reachable in time $t \in\left[t_{1}, t_{2}\right]$ is defined as:

$$
R_{\left[t_{1}, t_{2}\right]}^{-}\left(X_{f}\right) \nRightarrow \bigcup_{t \in\left[t_{1}, t_{2}\right]} R_{t}^{-}\left(X_{f}\right)
$$

Given a convex polyhedral set $X_{f}$ of initial states and a bound $t_{f}$ for the time, our objective is to compute a polyhedral approximation $\hat{R}_{\left[0, t_{f}\right]}^{-}\left(X_{f}\right)$ of the set $R_{\left[0, t_{f}\right]}^{-}\left(X_{f}\right)$, such that:

$$
\hat{R}_{\left[0, t_{f}\right]}^{-}\left(X_{f}\right) \subseteq R_{\left[0, t_{f}\right]}^{-}\left(X_{f}\right)
$$

To approximate the backward reachable set $R_{\left[0, t_{f}\right]}^{-}\left(X_{f}\right)$ we divide it into segments, each of which corresponds to a time interval. The $\mathrm{k}^{\text {th }}$ segment corresponding to the interval $\left[t_{k-1}, t_{k}\right]$ is the set $R_{\left[t_{k-1}, t_{k}\right]}^{-}\left(X_{f}\right)$. Then we approximate each segment separately and independently by a convex polyhedral set 
$\hat{R}_{\left[t_{k-1}, t_{k}\right]}^{-}\left(X_{f}\right)$ such that $\hat{R}_{\left[t_{k-1}, t_{k}\right]}^{-}\left(X_{f}\right) \subseteq R_{\left[t_{k-1}, t_{k}\right]}^{-}\left(X_{f}\right), \forall k$ Clearly:

$$
\hat{R}_{\left[0, t_{f}\right]}^{-}\left(X_{f}\right) \equiv \bigcup_{\forall k} \hat{R}_{\left[t_{k-1}, t_{k}\right]}^{-}\left(X_{f}\right) \subseteq R_{\left[0, t_{f}\right]}^{-}\left(X_{f}\right)
$$

We describe now how to obtain the set $\hat{R}_{\left[t_{k-1}, t_{k}\right]}^{-}\left(X_{f}\right)$. Let $R_{t_{k-1}}^{-}\left(X_{f}\right)$ be the reachable set at time $t_{k-1}$ and let us assume that it is convex polyhedral. Then the reachable set $R_{t_{k}}^{-}\left(X_{f}\right)$ is obtained from $R_{t_{k-1}}^{-}\left(X_{f}\right)$ by applying the affine transformation $y=T x+v$, where $T=e^{-A\left(t_{k}-t_{k-1}\right)}$ and $v=-\int_{t_{k-1}}^{t_{k}} e^{-A(t-\tau)} b d \tau$. Bearing in mind that convexity is preserved under affine transformations, we infer that the set $R_{t_{k}}^{-}\left(X_{f}\right)$ is a convex polyhedral set too. We define the set $C=\operatorname{conv}\left(R_{t_{k-1}}^{-}\left(X_{f}\right) \cup R_{t_{k}}^{-}\left(X_{f}\right)\right)$, where $\operatorname{conv}(\cdot)$ is the convex hull operator. $C$ is neither an over nor an under-approximation of $R_{\left[t_{k-1}, t_{k}\right]}^{-}\left(X_{f}\right)$. In order to become an under approximation, we have to "push" each of the faces of $C$ inwards by an appropriate amount $d \geq 0$. For that reason we define the operator $\operatorname{shrink}(C, d)$.

Definition 7 Let $C$ be a convex polyhedron

$$
\begin{array}{r}
C=\bigcap_{i=0}^{m_{f}}\left\{x \mid\left\langle a_{i}, x\right\rangle \leq b_{i}\right\}, \text { and let } d \in \Re^{+} . \text {We define: } \\
\operatorname{shrink}(C, d) \equiv \bigcap_{i=0}^{m_{f}}\left\{x \mid\left\langle a_{i}, x\right\rangle \leq b_{i}-d \cdot\left\|a_{i}\right\|\right\}
\end{array}
$$

We obtain a sufficient pushing amount $d$ by bounding the Hausdorff distance $h_{\{}\left(R_{\left[t_{k-1}, t_{k}\right]}^{-}\left(X_{f}\right), C\right)$. For a thorough definition of the Hausdorff metric $h(\cdot)$ and its properties see [21].

Lemma 1[21] Given sets $X_{1}, X_{2}, Y_{1}, Y_{2}$ in $\Re^{n}$, if $h\left(X_{1}, Y_{1}\right) \leq \varepsilon$ and $h\left(X_{2}, Y_{2}\right) \leq \varepsilon$ then $h\left(X_{1} \cup X_{2}, Y_{1} \cup Y_{2}\right) \leq \varepsilon$.

We estimate $h\left(R_{\left[t_{k-1}, t_{k}\right]}^{-}\left(X_{f}\right), C\right):$ The distance between an arbitrary point $x_{f} \in R_{t_{k-1}}^{-}\left(X_{f}\right)$ and the point $x\left(t, x_{f}\right)$ for $t \in\left[t_{k-1}, t_{k}\right]$ satisfies the inequality:

$$
\begin{aligned}
& \left\|x\left(t, x_{f}\right)-x_{f}\right\|=\left\|e^{-A t} x_{f}-\int_{t_{k-1}}^{t_{k}} e^{-A(t-\tau)} b d \tau-x_{f}\right\| \\
& \leq\left\|e^{-A t} x_{f}-x_{f}\right\|+\left\|\int_{t_{k-1}}^{t_{k}} e^{-A(t-\tau)_{b d}}\right\| x_{f} \|
\end{aligned}
$$

Considering a "short" time interval $\left[t_{k-1}, t_{k}\right]$ and after some algebraic manipulation we obtain:

$$
\left\|x\left(t, x_{f}\right)-x_{f}\right\| \leq M\left\|e^{-A \delta_{k}-I \|}+\sum_{i=0}^{\infty} \frac{\|A\|^{i}}{(i+1) !} \delta_{k}^{i}\right\| b \|
$$

where $M$ is a constant bounding $x_{f}$ and $\delta_{k}=t_{k}-t_{k-1}$. From lemma 1 it follows:

$$
h\left(R_{\left\{\bar{t}_{k-1}, t_{k}\right]}\left(X_{f}\right), R_{t_{k-1}}^{-}\left(X_{f}\right)\right) \leq M\left\|e^{-A \delta_{k}-I \|}+\sum_{i=0}^{\infty} \frac{\|A\|^{i}}{(i+1) !} \delta_{k}^{i}\right\| b \|
$$

Since $C=\operatorname{conv}\left(R_{t_{k-1}}^{-}\left(X_{f}\right) \cup R_{t_{k}}^{-}\left(X_{f}\right)\right)$ it holds:

$$
h\left(R_{t_{k-1}}^{-}\left(X_{f}\right), C\right) \leq M\left\|e^{-A \delta_{k}-l \|}\right\| \sum_{i=0}^{\infty} \frac{\|A\|^{i}}{(i+1) !} \delta_{k}^{i}\|b\|
$$

Considering the triangle inequality we finally obtain the desired bound for the Hausdorff distance $h\left(R_{\left[t_{k-1}, t_{k}\right]}^{-}\left(X_{f}\right), C\right)$ :

$$
h\left(R_{\left[t_{k-1}, t_{k}\right]}^{-}\left(X_{f}\right), C\right) \leq 2\left(M\left\|e^{-A \delta_{k}-I \|}+\sum_{i=0}^{\infty} \frac{\|A\|^{i}}{(i+1) !} \delta_{k}^{i}\right\| b \|\right)=d
$$

Choosing $d$ as the pushing amount, we conclude that

$$
\hat{R}_{\left[t_{k-1}, t_{k}\right]}^{-}\left(X_{f}\right)=\operatorname{shrink}(C, d)
$$

Repeating the above procedure for all the segments, we obtain the desired approximate reachable set $\hat{R}_{\left[0, t_{f}\right]}^{-}\left(X_{f}\right)$. Also it is clear that each segment $\hat{R}_{\left[t_{k-1}, t_{k}\right]}^{-}\left(X_{f}\right)$ can be computed independently and in any order. This implies that we do not have error transfer from one segment to another, i.e. the approximation error can become arbitrarily small choosing arbitrarily short time intervals $\delta_{k}$.

\subsection{Linear Systems with Set Valued Disturbance}

We consider now linear systems $\dot{x}=A x+u$ with $A \in \Re^{n \times n}$ and set valued disturbance $u \in U$, where $U$ is compact and convex set in $\Re^{n}$. The introduction of the uncertainty in $u$ makes the computation of the segment $\hat{R}_{\left[t_{k-1}, t_{k}\right]}^{-}\left(X_{f}\right)$ different. The main difference stems from the fact that even if $R_{t_{k-1}^{-}}^{-}\left(X_{f}\right)$ is convex polyhedral, the set $R_{t_{k}}^{-}\left(X_{f}\right)$ is an arbitrary smooth convex set. Therefore, we need a polyhedral under-approximation $\hat{R}_{t_{k}}^{-}\left(X_{f}\right)$ in order to apply the approximation technique of the previous section. Varaiya in [20], proposes an approximation scheme to compute $\hat{R}_{t_{k}}^{-}\left(X_{f}\right)$, based on the Maximum Principle of optimal control.

Lemma 2[20] Let $\left\langle c_{i}, x\right\rangle \leq \gamma_{i}^{*}$ be hyperplanes supporting $R_{t_{k-1}}^{-}\left(X_{f}\right)$ at $x_{i}^{*}, i=1, \ldots, \mu$. Let $x_{i}^{*}(s), \lambda_{i}^{*}(s), u_{i}^{*}(s), 0 \leq s \leq t$, be solutions to the following three equations 


$$
\left.\begin{array}{c}
\dot{x}_{i}^{*}(s)=-A x_{i}^{*}(s)-u_{i}^{*}(s), x^{*}(0)=x_{i}^{*} \\
\dot{\lambda}_{i}^{*}(s)=A^{T} \lambda_{i}^{*}(s), \lambda_{i}^{*}(0)=c_{i} \\
u_{i}^{*}(s) \in \arg \max \left\{\left\langle\lambda_{i}^{*}(s),-A x_{i}^{*}(s)-u\right\rangle \mid u \in U\right\}
\end{array}\right\}
$$

Let $\gamma_{i}^{*}(s)=\left\langle\lambda_{i}^{*}(s), x^{*}(s)\right\rangle$. Then:

$\hat{R}_{t_{k}}^{-}\left(X_{f}\right)=\operatorname{conv}\left(x_{1}^{*}(s), \ldots, x_{\mu}^{*}(s)\right) \subset R_{t_{k}}^{-}\left(X_{f}\right)$.

Next, we estimate an appropriate amount, by which $C=\operatorname{conv}\left(R_{t_{k-1}}^{-}\left(X_{f}\right) \cup \hat{R}_{t_{k}}^{-}\left(X_{f}\right)\right)$ should be shrunk. For every signal $u \in U$, we define its mean value $u_{m}$ in the time interval $\left[t_{k-1}, t_{k}\right]$ as:

$$
u_{m}=\frac{1}{\delta_{k}} \int_{t_{k-1}}^{t_{k}} u(\tau) d \tau
$$

Lemma 3[4] Let $\tilde{R}_{\left[t_{k-1}, t_{k}\right]}^{-}\left(X_{f}\right)$ be the reachable set

$R_{\left[t_{k-1}, t_{k}\right]}^{-}\left(X_{f}\right)$ under constant disturbance signal $u_{m}$, as defined in (14). Then $\forall u \in U$ and $\forall t \in\left[t_{k-1}, t_{k}\right]$ it holds

$$
h\left(R_{\left[t_{k-1}, t_{k}\right]}^{-}\left(X_{f}\right), \tilde{R}_{\left[t_{k-1}, t_{k}\right]}^{-}\left(X_{f}\right)\right) \leq 2 M_{u} \delta_{k}^{2} e^{\left\|A \delta_{k}\right\|}
$$

where $M_{u}$ is a constant bounding $\|u\|$.

Employing the results of the previous subsection, lemma 3 and the triangle inequality, we obtain:

$$
\begin{aligned}
& h\left(R_{\left[t_{k-1}, t_{k}\right]}^{-}\left(X_{f}\right), C\right) \leq \\
& h\left(R_{\left[t_{k-1}, t_{k}\right]}^{-}\left(X_{f}\right), \tilde{R}_{\left[t_{k-1}, t_{k}\right]}^{-}\left(X_{f}\right)\right)+h\left(\tilde{R}_{\left[t_{k-1}, t_{k}\right]}^{-}\left(X_{f}\right), C\right) \Rightarrow \text {. } \\
& h\left(R_{\left[t_{k-1}, t_{k}\right]}^{-}\left(X_{f}\right), C\right) \leq
\end{aligned}
$$

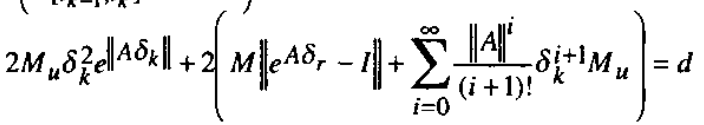

Consequently, choosing $d$ as the new pushing amount, we obtain an under approximation of the set $R_{\left[t_{k-1}, t_{k}\right]}^{-}\left(X_{f}\right)$ for the considered class of systems as $\hat{R}_{\left[t_{k-1}, t_{k}\right]}^{-}\left(X_{f}\right)=\operatorname{shrink}(C, d)$.

Unlike the case of affine systems, lemma 2 implies that we have error propagation from one segment to another. Therefore, we cannot consider each segment $\hat{R}_{\left[t_{k-1}, t_{k}\right]}^{-}\left(X_{f}\right)$ independently. Besides, each segment has to be computed sequentially. Finally, it is clear that the approximation error grows with time. So in order to keep it in a desired level, both the time intervals $\left[t_{k-1}, t_{k}\right]$ and the time bound $t_{f}$ must be relatively "small".

\section{Application to a Batch Control Problem}

We have applied the design algorithm of section 3 to a simplified batch control problem. We consider the chemical-batch reactor illustrated in figure 2. A similar version of this reactor has been used in [7] for verification purposes.
First, a certain amount of dissolved substance $B$ is fed into the reactor by opening valve $v_{B}$. After switching on the stirrer motor, a solution of substance $\mathrm{A}$ is added through valve $v_{A}$ until its concentration $c_{A}$ reaches an upper limit $\left(0.6 \mathrm{Kmole} / \mathrm{m}^{3}\right)$.

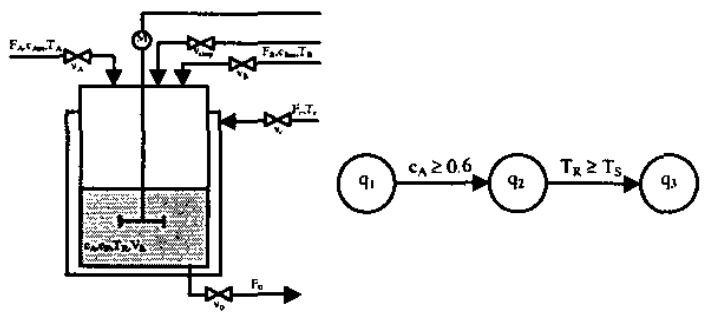

Figure 2: The Reactor and its Automaton Representation

Then the exothermic reaction $A+B \rightarrow D$ starts properly. As a result, the temperature $T_{R}$ inside the reactor rises. To avoid a dangerous situation, the cooler must be switched on at an appropriate instant during the warm-up phase of the reaction. The cooler supplies the reactor with a cooling fluid through valve $v_{c}$. We denote its flow and its temperature by $F_{c}$ and $T_{C}$. When the product concentration $c_{D}$ reaches the desired value $\left(0.3 \mathrm{Kmole} / \mathrm{m}^{3}\right)$, the reactor is drained by opening the valve $v_{0}$ and the stirrer is switched off. In case of emergency, due to excessive temperature $(310 K)$, a stopper liquid can be discharged into the vessel by opening the valve $v_{\text {stop. }}$ On the other hand, when the temperature drops below a certain value $(283 K)$ the reaction fails and the reactor must be drained through valve $v_{0}$. Our control objective is to choose the temperature $T_{S}$, during the warm-up phase, that the cooler must be switched on so that the product reaches the desired concentration, avoiding overheating or overcooling of the reactor. We express this batch control problem as a target control problem on the hybrid automaton of figure 2 :

In location $q_{1}$ substance $\mathrm{A}$ is fed in the reactor, in $q_{2}$ the reaction warms up, while in location $q_{3}$ the cooler is switched on. The continuous dynamics in each location $q_{i}, i=\{1,2,3\}$ is given by $\dot{x}=A_{q} x+b_{q}$, where $x=\left[c_{A}, c_{D}, T_{R}, V_{R}\right]^{T}$ and

$\left.A_{1}=\left[\begin{array}{cccc}-0.0008 & 0 & 0 . & -0.003 \\ 0 . & -0.0008 & -0 . & 0 . \\ 0.0003 & 0 & -0.0022 & -0.0113 \\ 0 & 0 & 0 & 0\end{array}\right] \quad b\right]=\left[\begin{array}{c}-0.0052 \\ 0.0062 \\ 0.663 \\ 0.0008\end{array}\right]$

$A_{2}=\left[\begin{array}{cccc}-0.0001 & 0 & 0.0002 & 0 \\ 0.0001 & 0 & -0.0001 & 0 \\ 0.0037 & 0 & -0.0051 & 0 \\ 0 & 0 & 0 & 0\end{array}\right] \quad b_{2}=\left[\begin{array}{c}-0.0491 \\ 0.0246 \\ 1.6136 \\ 0\end{array}\right]$

$A_{3}=\left[\begin{array}{cccc}-0.0001 & 0 & 0 & 0 . \\ 0 . & 0 & 0 & -0 . \\ 0.0018 & 0 & -0.0073 & 0 \\ 0 & 0 & 0 & 0\end{array}\right] \quad b_{3}=\left[\begin{array}{c}-0.0016 \\ 0.0008 \\ 2.0857 \\ 0\end{array}\right]$ 
$V_{R}$ denotes the volume of the fluid in the reactor. The initial set $l=q_{1} \times X_{1}$ and the target set $F=q_{3} \times X_{3}$ are defined by:

$$
X_{1}=\left\{\begin{array}{l}
0 \leq c_{A} \leq 0.01 \mathrm{Kmole} / \mathrm{m}^{3} \\
0 \leq c_{D} \leq 0.01 \mathrm{Kmole} / \mathrm{m}^{3} \\
290 \leq r_{R} \leq 291 \mathrm{~K} \\
1 \leq v_{R} \leq 1.01 \mathrm{~m}^{3}
\end{array} \quad X_{3}=\left\{D \geq 0.3 \mathrm{Kmole} / \mathrm{m}^{3}\right\}\right.
$$

The transition $\left(q_{1}, q_{2}\right)$ is uncontrollable with

$G\left(q_{1}, q_{2}\right): c_{A} \geq 0.6 \mathrm{Kmole} / \mathrm{m}^{3}$, while for the controllable transition $\left(q_{2}, q_{3}\right)$ it holds $G\left(q_{2}, q_{3}\right): 300 \leq T_{R} \leq 310 \mathrm{~K}$. The invariant set for every $q_{i}, i=\{1,2,3\}$ is defined as:

$$
I N V\left(q_{i}\right)=\left\{\begin{array}{l}
0 \leq c_{A} \leq 0.6 \mathrm{Kmole} / \mathrm{m}^{3} \\
0 \leq c_{D} \leq 0.4 \mathrm{Kmole} / \mathrm{m}^{3} \\
283 \leq T_{R} \leq 310 \mathrm{~K} \\
1 \leq V_{R} \leq 1.5 \mathrm{~m}^{3}
\end{array}\right.
$$

Applying the control synthesis algorithm to the problem, we obtain the control guard $G^{*}\left(q_{2}, q_{3}\right)$, depicted in figure 3 . Finally, the desired switching temperature $T_{S}$ is given by:

$$
T_{S}=\left\{x_{3}: x \in G^{*}\left(q_{2}, q_{3}\right)\right\}
$$

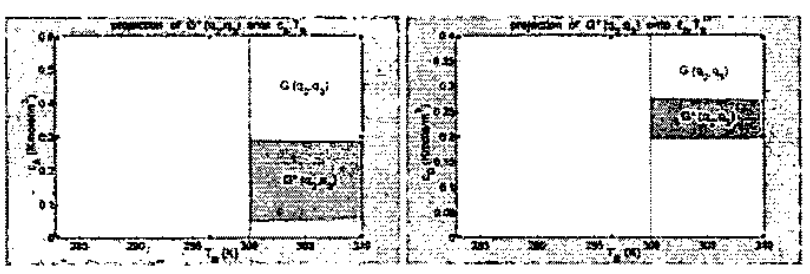

Figure 3: 2D Representation of $G^{*}\left(q_{2}, q_{3}\right)$

\section{Conclusion}

Although the results of our approach are very satisfactory at this early stage, there is plenty of room for further research and improvements. The proposed approximate reachability analysis is rather conservative leading to large approximation enors. Improved under-approximation schemes for systems with linear dynamics should be further studied. Also, under-approximations of reachable sets for non-linear continuous dynamics are needed for extension of this work to more practical systems. Finally, optimal target control with respect to both continuous and discrete dynamics is another extension that deserves investigation in a future work.

\section{Acknowledgement}

This work has been supported by the ESPRC under grant: GR/M51260.

\section{References}

[1] E.Asarin, O.Bournez, T.Dang, O. Maler: Approximate Reachability Analysis of Piecewise-Linear Systems. Hybrid Systems: Computation and Control, LNCS 1790, Springer, 2000. [2] O.Botchkarev, S.Tripakis: Verification of Hybrid Systems with Linear Differential Inclusions Using Ellipsoidal Approximation. Hybrid Systems: Computation and Control, LNCS 1790, Springer, 2000.

[3] A.Chutinan, B.Krogh: Computing Polyhedral Approximations to Flow Pipes for Dynamic System. IEEE Conference on Decision and Control, 1998.
[4] T.Dang: Verification and Synthesis of Hybrid Systems. Ph.D. Thesis, VERIMAG, Grenoble, 2000.

[5] T. Dang, O.Maler: Reachability Analysis via Face Lifting. Hybrid Systems: Computation and Control, LNCS 1386, Springer, 1998.

[6] M.Greenstreet, I. Mitchelf: Reachability Analysis using Polygonal Projections. Hybrid Systems: Computation and Control, LNCS 1569, Springer, 1999.

[7] S.Kowalewski, S.Engell, O.Stursberg: Verification of Logic Controllers for Continuous Plants. Advances In Control, Highlights of ECC 1999, Springer 1999.

[8] S.Kowalewski, O.Stursberg: The Batch Evaporator: A Benchmark Example for Safety Analysis of Processing Systems under Logic Control. (WODES '98).

[9] S.Kowalewski, O.Stursberg, M.Fritz, H.Graf, I.Hoffman, J.Preussig, M.Remelhe, S.Simon, H.Treseler: A Case Sstudy in Tool-Aided Analysis of Discretely Controlled Continuous Systems: The Two-Tank Problem. Hybrid Systems V, LNCS, Springer, 1998.

[10] A.Kurzhanski, P.Varaiya: Ellipsoidal Techniques for Reachability Analysis. Hybrid Systems: Computation and Control, LNCS 1790, Springer, 2000.

[11] A.Kurzhanski, P.Varaiya: Ellipsoidal Techniques for Reachability Analysis. Internal Approximation. Technical Report, 1998.

[12] B.Lenartson, M.Tittus, B.Egardt, S.Petterson: Hybrid Systems in Process Control. IEEE Control Systems Magazine, October 1996.

[13] E.Martins, M.Pascoal, J.Dos Santos: Labelling Algorithms for Ranking Shortest Paths. CISUC Technical Report, Department of Mathematics, Coimbra University, Portugal, 2000.

[14] E.Martins, M.Pascoal, J.Dos Santos: The K Shortest Paths Problem. CISUC Technical Report, Department of Mathematics, Coimbra University, Portugal, 1998.

[15] G.Pappas: Hybrid Systems: Computation and Abstraction. $\mathrm{Ph} . \mathrm{D}$. Thesis, University of Califomia at Berkeley, 1998.

[16] J.Preussig, O.Stursberg, S.Kowalewski: Reachability Analysis of a Class of Switched Continuous Systems by Integrating Rectangular Approximation and Rectangular Analysis . Hybrid Systems: Computation and Control, LNCS 1569, Springer, 1999. [17] O.Shakemia, G.Pappas, S.Sastry: Semidecidable Synthesis for Triangular Hybrid Systems. Hybrid Systems: Computation and Control, LNCS 2034, Springer, 2001.

[18] M.Tittus, B.Egardt: Control Design for Integrator Hybrid Systems: IEEE Transactions on Automatic Control: Special Issue on Hybrid Systems, 43(4):491-500, April 1998.

[19] A.Trontis, M.Spathopoulos: Target control for Linear Hybrid Systems. ECC 2001.

[20] P.Varaiya: Reach Set Computation Using Optimal Control. In Proc. KIT Workshop, VERIMAG, Grenoble, 1998.

[21] R.Webster: Convexity. Oxford University Press, 1994. 